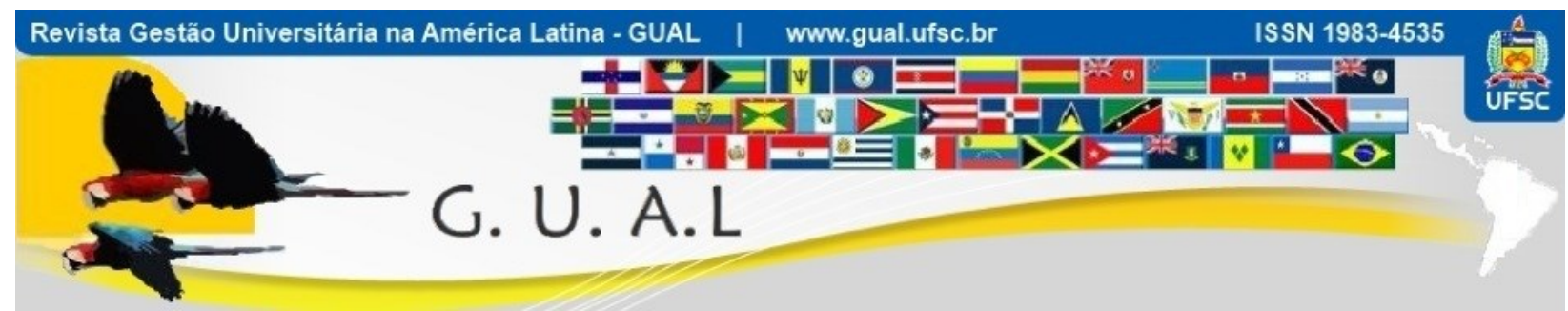

DOI: http://dx.doi.org/10.5007/1983-4535.2013v6n4p37

\title{
GLOBALIZACIÓN DE LA EDUCACION SUPERIOR EN PARAGUAY
}

\author{
GLOBALIZATION OF HIGHER EDUCATION IN PARAGUAY
}

Hugo Alfredo Recalde, Licenciado

Universidad Nacional de Caaguazú - UNC@ hugo_re@hotmail.com

Willian Cantero Lusardi, Mestre Universidad Nacional de Caaguazú - UNC@ williancantero@gmail.com

Juan Angel Jara Acosta, Licenciado Universidad Nacional de Caaguazú - UNC@ jaraju01@hotmail.com

Recebido em 29/novembro/2013

Aprovado em 29/novembro/2013

Sistema de Avaliação: Double Blind Review

Esta obra está sob uma Licença Creative Commons Atribuição-Uso. 


\title{
RESUMEN
}

El inicio del desarrollo e innovación del sistema de Educación Superior en Paraguay nació en el año 1889 con la creación de la Universidad Nacional de Asunción, siendo la primera universidad Nacional en el país y en 1960 la Universidad Católica Nuestra Señora de la Asunción, la primera universidad privada; desde entonces fue postergada la creación de Universidades en Paraguay por más de 100 años, causa el sistema de gobierno político implementado en aquel entonces, al no haber centro de estudios especializados también se postergo el desarrollo intelectual de las personas, desde 1989 con la caída del sistema dictatorial se han abierto un abanico de posibilidades con la apertura de universidades privadas y públicas. Actualmente el Paraguay cuenta con 52 universidades en pleno funcionamiento de las cuales, 8 son universidades públicas y 44 de administración privada, a esto podemos agregar un total de 11 carreras acreditadas a nivel MERCOSUR, certificaciones otorgadas mediante el Sistema de Acreditación Regional de Carreras Universitarias (ARCUSUR), por la Agencia Nacional de Evaluación y Acreditación de la Educación Superior (ANEAES). Es evidente el cambio cuali - cuantitativo experimentado por el sistema de Educación Superior en Paraguay, acompañando de la mano a las tecnologías de la información y la educación que hacen una Globalización eficaz y eficiente del conocimiento.

Palabras-clave: Educación Superior. Globalización Educativa. Evolución de la E. S.

\begin{abstract}
The development of the higher education in Paraguay begins in 1889, with the opening of the National College of Asunción. In the years of dictatorship the Paraguayan people could not study in the universities. Although in Paraguay, exist the law that support the university education, the separation among the different levels of the educational system difficult the admission to the college. There are 54 colleges in Paraguay (46 private and 8 public schools), there are 288 faculties that teach 1633 degree course. Although the education in Paraguay has been change since 1989 and the college accepts some students, the most college are private yet, the educational system in these universities is unsatisfactory, and the training of the students is deficient, because these universities are more interested in the income that in the teaching. Colleges either finance scientific projects. On the other hand the university is a way to reproduce the knowledge and not to produce knowledge for the development of the country. The college are lack of laboratories to the investigation. The university system needs a higher budget, most cooperation, a better system of admission; consolidate the technic course, more communication among the different universities and most cooperation with national and international institutions.
\end{abstract}

Keywords: Higher Education. Admission. Unsatisfactory. Scientific Projects. Higher Budget. 


\section{CARACTERISTICAS PRINCIPALES DEL SISTEMA DE EDUCACION EN PARAGUAY}

La educación superior en Paraguay se inició en 1889 con la creación de la Universidad Nacional de Asunción, la primera en el país, en 1960 la primera universidad privada, Universidad Católica Nuestra Señora de la Asunción, a partir de ahí después de 1989 se dio la expansión masiva de universidades por sobre todo de carácter privado.

Para empezar a entender el sistema educativo y por sobre todo en Educación Superior es importante destacar la ley en educación superior que rige actualmente en el Paraguay es la ley 4995/13 de universidades, el objeto de la ley es regular la educación superior en el Paraguay como parte del sistema educativo, pero debemos afirmar que un déficit de la educación y que en muchos países latinoamericanos acontece es la separación de las partes, como la educación inicial por un lado, la secundaria y técnica por otro y las Universidades e institutos por otro lado, esto hace que sea muy dificultoso el ingreso en las universidades de los jóvenes que terminan el proceso secundario.

Actualmente existen 54 universidades en el Paraguay, que desarrollan un mínimo de tres áreas del saber, de los cuales 8 son de carácter oficial y 46 privadas, con 288 facultades que ofertan 1633 carreras. (Datos actualizados a abril de 2013), existen 38 Institutos Superiores que se desempeñan en un solo campo del saber, de los cuales 8 son oficiales y 30 del sector privado, en tanto 389 Institutos profesionales; 253 son institutos técnicos superiores; 10 oficiales y 243 privados.

En referencia a lo expuesto, 136 son institutos de formación docente en dónde; 40 son oficiales y 96 privados, conforme el livro del José Nicolás Morinigo, DATOS SOBRE EDUCACION SUPERIOR EN PARAGUAY.

Se desea resaltar el cambio cuali - cuantitativo y de conocimientos múltiples en las diferentes áreas del saber que denota la evolución de la educación superior en Paraguay, en cuanto al crecimiento de las instituciones a partir de 1989, en donde se sucedió el cambio de gobierno a la democracia en el Paraguay; evidentemente este sistema de gobierno conlleva un amplio margen de divulgación de los conocimientos múltiples a los habitantes de este país, no asegurando por un lado un desarrollo pleno de las capacidades de las personas ni tampoco la obtención de un puesto de trabajo por haber estudiado en alguna de las universidades del Paraguay por distintos motivos que entraremos a detallar.

Vemos que el Paraguay ha vivido un sistema de autoritarismo desde prácticamente 1811 hasta 1989, recién con la caída del Stronissmo que representa uno de los gobiernos 
dictatoriales más álgidos en la historia del Paraguay, y que en cierta medida y a opinión de algunos destacados sociólogos paraguayos como Cardozo, ha formado una cultura sumisa y estoica en la ciudadanía paraguaya, en el año 1989 que se experimentó el inicio del proceso democrático, con ello se pudo dar oportunidades a los jóvenes de poder estudiar en las universidades y formarse como profesional, años anteriores a 1989, solo una elite exclusiva de algunas personas podían acceder a las universidades paraguayas, ahora bien hay que ser sinceros que también se han creado universidades de carácter garaje que significan universidades comerciales que tienen por objetivo lucrar con la enseñanza superior, ya que existen los que tienen clases dos o tres veces por semana hasta una vez por semana (sábados cuatro horas).

Entendemos que esto significa una mentira al joven que invierte tiempo y dinero para asistir a la universidad y salir de ella sin las capacidades necesarias para afrontar la vida profesional, también tenemos el caso de personas de otros países que llegan a Paraguay a los efectos de estudiar en universidades de carácter privado y lo hacen una vez en un año aprovechando las vacaciones de quince días y en otras las vacaciones de verano, lo cual creemos como hemos dicho que se realizan en carácter lucrativo y no con miras a formar profesionales de excelencia. No se considera que el tiempo en aula sea de carácter determinante en ello, pero si la preparación de programas, mallas curriculares que deben estar actualizadas y por sobre todo la implementación de los contenidos de estas mallas tanto como la práctica de la extensión universitaria, que no es más que la práctica de los actos que hacen los profesionales para mejorar su calidad profesional en el área que le toque desempeñar.

Tampoco las Universidades de carácter nacional pueden darnos la entera certeza de que se está formando profesionales de calidad, esto también constituye una llamada de atención a efectos de que se pueda ir soslayando problemas comunes que existen en las universidades nacionales, que son rubros de docentes, postulación a cargos sin concursos en muchos casos, docentes que no cumplen con el rol, la falta de investigación y extensión que pueda infundir en el alumno la práctica de su profesión en su área.

Otro de los elementos interesante y obligados es la investigación científica que hace que los alumnos ayudados por docentes universitarios puedan buscar soluciones positivas, desde el punto de vista de la ciencia para problemas que susciten en la sociedad y el campo laboral, dentro del modelo siempre de la universidad local; la especialización en estas ciencias es aún muy escasa por factores económicos en el área de la investigación, también podríamos nombrar la falta de gestión de la universidad para poder acceder a fondos de empresas 
interesadas en conocimientos certeros para realizar inversiones sobre proyectos realizados por la universidad y desde luego que estas inversiones sean exitosas.

Un aspecto fundamental a tener en cuenta es la expansión de la educación superior a partir del 1989, ha logrado la masificación de la misma, sin lugar a dudas un logro importante, pero bajo el enfoque de la transmisión y reproducción de conocimiento como modelo académico por elexelencia, esto en total detrimento a los dos pilares fundamentales de la educación superior como lo son la extensión y la investigación esta situación conlleva a que los profesionales graduados en las distintas casas de estudio desconocen la esencia y la importancia que tiene la articulación de la academia, la extensión y la investigación como un modelo que genere un desarrollo sustentable de las comunidades y de las regiones siendo estos profesionales los que los dominantes de las demandas de docentes que genera la mencionada expansión, convirtiéndose en un ciclo vicioso lejos de los postulados de la reforma de Córdoba por citar solo una, el cual debería ser el norte de toda institución de educación superior universitaria.

Es importante abordar los factores positivos como negativos que tiene la Educación Superior en Paraguay, que depende en cierta manera de los momentos políticos que sufre el País, de acuerdo a los gobernantes de turno suben representantes como Ministros de Educación los cuales van cambiando las expectativas no sustentando un plan estratégico vigente en el Ministerio de Educación y Cultura, se ha creado un sistema de Evaluación y Acreditación de las Universidades (ANEAES), el cual no han podido brindar sus frutos por razones políticas, falta de presupuesto o simplemente por no contar con un cuerpo colegiado para el desempeño de sus funciones; otro de los aspectos que creemos es una debilidad es la implementación de la investigación en Paraguay.

Según el Consejo de Ciencia y Tecnología del Paraguay (CONACYT), somos la región que menos investigaciones brinda en la región, el Paraguay invierte cerca del 4\% del Producto Interno Bruto, que en la nueva la ley 4995 del 2013, hay una cierta esperanza que se invierta hasta el $7 \%$. para educación, esto significa que las universidades no cuentan con rubros para investigadores ni para programas que puedan incentivar a investigadores novatos, existen unos fondos concursables actualmente que son para investigadores máster o grupos de investigadores pagados por sectores privados con experiencia calificada, quedando así los del sector público sin mucho que hacer, tampoco las universidades especialmente de carácter público y ni que hablar las de carácter privado, cuentan con laboratorios de investigación por el problema de rubros haciendo esta tarea todavía más dificultosa. 
Siguiendo esta línea de análisis sobre los aspectos negativos de la educación superior en el Paraguay es la inculturación de la Extensión Universitaria, la disociación de la academia y la investigación atrasan el desarrollo de los pueblos, del total de las universidades mencionadas al inicio del documentos la extensión universitaria está inserta en la carta orgánica de las universidades nacionales que solo son 8 y estas incluyen programas de extensión universitaria en el devenir de las vidas de los estudiantes, de la cual solo 1 cuenta con presupuesto del estado paraguayo para el desarrollo de los programas mencionados, esto debería ser un puntal de análisis y profundo debate sobre la importancia de la función social de la universidad.

Esto pregonado en la reforma de Córdoba del 1918, el fortalecimiento de la función social de la Universidad, vía proyección de su quehacer a la sociedad mediante los programas de extensión universitaria y defunción cultural, este prometedor cometido, capaz de vincularla más estrechamente con la sociedad y sus problemas, de volcarla hacia su pueblo, haciendo a este participe de su mensaje y transformándose en su conciencia cívica y social. Contrastar esto con la realidad y las voluntades políticas de los gobernantes de turno, resulta una triste realidad, que en el caso de algunas minorías (entiéndase universidades) son afrontadas con mucha creatividad e innovación, además de una alta dosis de autogestión, a los efectos de llevar a cabo los programas de extensión universitaria y cultivar esta semilla, para convertirse en un centro por excelencia para el estudio científico de los grandes problemas regionales, nacionales e internacionales, para cosechar el desarrollo de los pueblos.

Pensar en laboratorios de investigación seria un adelanto demasiado interesante, debemos tener en cuenta de igual manera que ni siquiera las universidades públicas, a acepción de algunas como la UNA y la UCA entre otras minorías, cuentan con biblioteca física acorde a las necesidades y las carreras que estas ofrecen a los alumnos.

La informatización o las tecnologías de la información y la comunicación, de igual manera constituyen una opción importante ante el problema antes expuesto, es necesario ir capacitando a los jóvenes y por sobre todo a los docentes universitarios en el uso de las nuevas tecnologías de la información y la comunicación a los efectos de poder dar respuestas acordes a los tiempos, conocemos de la gran capacidad de los jóvenes llamados nativos virtuales que tienen acceso irrestricto a las informaciones actuales en el país y el mundo, sabemos que no todo está escrito en los libros y lo que está escrito tarda tiempo en ser editado, así corremos el riesgo que cuando se lanza la información en los libros en la realidad ya ha sido sustituida, esta es una de las ventajas en la rapidez de la información, y desventajas a los 
que escriben libros, acotando que no todo es la tecnología ni el internet, sino que esto constituye una manera de acompañar el proceso de evolución científica y tecnológica por el cual está pasando el conocimiento en el mundo; podemos hacer de las clases interactivas y de fuerte atención a los jóvenes trayendo informaciones nuevas e innovadoras teniendo en cuenta simplemente de la manera que se presentan estas informaciones.

Los pilares que sustentan las universidades en el Paraguay y son la academia, la investigación y la extensión algunos que otras universidades hablan del desarrollo y hoy también se abarca un tema de gran importancia que es la Responsabilidad Social Universitaria, que creemos son temas de vital importancia a la hora de hacer universidad, de eso nos ocuparemos para tratar de dar algunos puntos de vista al respecto.

Actualmente la función del docente no es enseñar, sino servir al joven de guía para que este adopte el proceso de enseñanza aprendizaje, esto denota los cambios holísticos que suceden en la educación superior, no perdiendo de vista la necesidad a que los docentes vayan capacitándose en el ámbito de la educación, sabemos que un profesional de carreras técnicas como contabilidad e ingeniería tiene conocimiento y cuenta con capacidades, pero debemos tener en cuenta que debe estar formado en pedagogía o en el arte de poder enseñar, existen docentes en el Paraguay que son excelentes como profesionales en su ámbito, pero que no pueden llegar a tener éxito en las ciencias de la educación, consideramos imprescindible que los profesionales sean formados en la educación ya que no hay que olvidar que la universidad es un centro especializado en multiplicidad de conocimientos pero que al final su objetivo principal es la formación de personas versadas en la profesión, si no es así puede que el joven fracase por motivos ya expuestos.

Otro de los temas ampliamente debatido en las universidades paraguayas es la responsabilidad social actualmente, también está enfocado desde el punto de vista de la extensión universitaria, y la pregunta de muchos es si ¿esto o no un compromiso de las universidades públicas y privadas?, en este sentido hay que entender que muchas de las universidades privadas no tienen como eje normativo la extensión universitaria ya que está considerado como una función y más aún compromiso de universidades de carácter oficial, ya que son las universidades en donde no se abona cuotas, si matricula y se entiende que el estado es quien paga el estudio del alumno o alumna, siendo como una devolución a la sociedad o comunidad por esa prestación que el estado invierte en el joven, realizando las universidades privadas intervenciones que se podrían considerar como filantropía, teniendo en cuenta que hay otras que si lo aplican de manera debida. La extensión universitaria como 
su nombre lo dice es extender los conocimientos profesionales de los alumnos, funcionarios y docentes hacia la sociedad por varios motivos que pasaremos a nombrar:

- La devolución de la inversión que realiza el estado paraguayo en los alumnos es en cierta medida uno de los temas interesantes que hay que abordar, ya que muchos alumnos estudian con becas del estado y los que no tienen facilidades económicas al estudiar en una universidad de carácter oficial, los docentes la infraestructura y otros costos pecuniarios los absorbe el estado paraguayo a los efectos que el joven pueda realizar sus estudios, a esta situación el joven debe devolver en cierta medida al estado lo que este invierte en el retornando socialmente a la comunidad con labores que denoten desarrollo de sus capacidades, por ejemplo un alumno de medicina que debe realizar atenciones básicas en comunidades carenciadas como detectar niños en riesgo de crecimiento y ayudar en la detección de parasitosis en ellos, un alumno de veterinaria realizar vacunaciones antirrábicas entre otras a los efectos de favorecer a la comunidad donde se está formando como profesional y las personas colaboran con ellos pagando sus impuestos.

- La práctica profesional del alumno a la comunidad, al realizar las acciones nombradas en el punto anterior es importante destacar que estos alumnos están actuando con conocimiento científico y afianzándose en su profesión.

No hay que olvidar que tanto la academia, la extensión y la investigación deben actuar de manera estructurada y coordinada, siendo estos elementos necesarios y ausentes en el sistema educativo paraguayo, de poder realizar estas actividades en conjunto se solucionarían varios problemas de la sociedad y cumpliríamos con una necesidad fundamental de las universidades de ser respuesta a los problemas sociales.

Un abordaje sobre la ley de educación superior, la base legal de la articulación de la universidad en el régimen legal del Paraguay, tras una centuria de experiencias y labor de la educación superior en el Paraguay, como una medida para orientar un nuevo rumbo de la educación superior es aprobada la LEY No 4995: Ley de educación superior, cuyo origen es la Secretaría del Viceministerio de Educación Superior, publicado en Asunción Paraguay, 02 de agosto de 2013. Una visión acorde a los nuevos tiempos y con una profunda filosofía de regularizar todos los aspectos negativos antes mencionados en este ensayo y sobre la cual están cifrados los encausamientos que deben realizar las autoridades tanto del nivel nacional como de las universidades para dar nuevos bríos a la educación superior universitaria de cara al siglo XXI, a la globalización y a la revolución del conocimiento propios del nuevo mundo en el cual vivimos.

En conclusión existen innumerables problemas en la Educación Superior tanto del Paraguay como de otros países, pero no queremos finalizar este ensayo sin antes nombrar que 
solo el $4.9 \%$ de los jóvenes que terminan la educación secundaria tienen acceso a universidades tanto públicas como privadas y solo el $70 \%$ de estos terminan la educación terciaria siendo una cifra alarmante teniendo en cuenta que muchos quedan por el camino.

En relación a los problemas expuestos, nombramos algunos desafíos necesarios en la Educación Superior en el Paraguay a los efectos de mejorar la educación para el presente y el futuro en Paraguay:

1. Mayor financiamiento para la Educación Superior en especial en el área de investigación y la extensión.

2. Docentes investigadores de medio tiempo o tiempo completo

3. Implementación adecuada de la RSU de las Universidades en la zona de acción (crecimiento eco socio económico y cultural).

4. Adecuarse a los estándares internacionales del MERCOSUR Educativo.

5. Realizar estudios de mercado, para la creación de carreras adecuadas a la realidad de cada región, con un sistema de elección vocacional sobre carreras universitarias.

6. Establecer las reformas adecuadas a las legislación de la Educación Superior.

7. Generar espacios de diálogo entre el sector político social y Educación Superior.

8. Buscar nuevos sistema de admisión para el examen de ingreso en la universidad, fortaleciendo las de las carreras técnicas como las ingenierías.

9. Implementar cursos de especialización de docentes en sus áreas de acción.

10. Desarrollo del sistema de información integrado externo e interno de las Universidades y entre las Universidades.

11. Fortalecer y dinamizar la agencia de acreditación para Educación Superior.

12. Crear Redes de Cooperación con organismos nacionales e internacionales

13. Flexibilizar los currículos de las Universidades con miras a la movilidad a nivel nacional e internacional.

\section{BIBLIOGRAFÍA}

APROXIAMACIÓN AL CONCEPTO DE RSU.

$<$ http://es.scribd.com/doc/72804658/aproxiamacion-al-concepto-de-rsu $>$ [consulta: 07 octubre 2013] 


\section{EDUCACION, APRENDIZAJE Y DESARROLLO EN UNA SOCIEDAD}

\section{MULTICULTURAL}

$<$ http://www.univalle.edu/publicaciones/brujula/brujula1/pagina13.htm $>$ [consulta 10 octubre 2013]

EL CONCEPTO DE UNIVERSIDAD.

$<$ http://www.anuies.mx/servicios/p_anuies/publicaciones/revsup/res102/txt3.htm $>$ [consulta]

FUNCIÓN SOCIAL DE LA UNIVERSIDAD.

$<$ http://www.houssay.org.ar/hh/discurso/univsoci.htm>[consulta: 09 octubre 2013]

FUNCIONES DE LA UNIVERSIDAD.

$<$ http://uasd.edu.do/website/detalles/general_funciones.htm>[consulta: 09 octubre 2013]

GREEN PAPER EUROPEAN TRANSPARENCY INITIATIVE.

$<$ http://europa.eu.int/comm/off/green/index_es.htm>[consulta 11 octubre 2013]

LA RESPONSABILIDAD SOCIAL DE LA UNIVERSIDAD EN LA PROMOCIÓN DEL CAPITAL SOCIAL PARA EL DESARROLLO SUSTENTABLE.

$<$ http://www.iigov.org/dhial/?p=46_05>[consulta 12 octubre 2013]

\section{LA UNIVERSIDAD EN AMERICA LATINA FRENTE A NUEVOS DESAFIOS}

POLÍTICOS, SOCIALES Y ACADEMICOS.

$<$ http://www.congresoretosyexpectativas.udg.mx/Congreso $\% 206 /$ Conferencias $\% 20$ Magistrale s/Mesa1/norbertofernandez.pdf $>$ [consulta: 10 octubre 2013].

\section{LA UNIVERSIDAD Y SUS FUNCIONES EN LA SOCIEDAD ACTUAL.}

$<$ http://www.ccee.edu.uy/investigacion/lineas/messina/tessinappt.pdfUniversidad.pdf $>$ [consul ta: 08 octubre 2013]

LEY No 4995. Ley de educación superior, Secretaría del Viceministerio de Educación Superior, Asunción Paraguay, 02 de agosto de 2013.

MORINIGO, José Nicolás, datos sobre la Educación Superior en el Paraguay, abril 2012

RAMA, Claudio, Nuevos modelos universitarios en América Latina, junio 2013

REFLEXION ACERCA DE LA FUNCION DE LA UNIVERSIDAD: LA INVESTIGACIÓN.

$<$ http://ciruelo.uninorte.edu.co/pdf/huellas/9/Huellas_9_6_ReflexionesAcercadelaFunciondela $>$ [consulta 08 octubre 2013] 


\section{ANEXOS}

\section{Cantidad de Universidades}

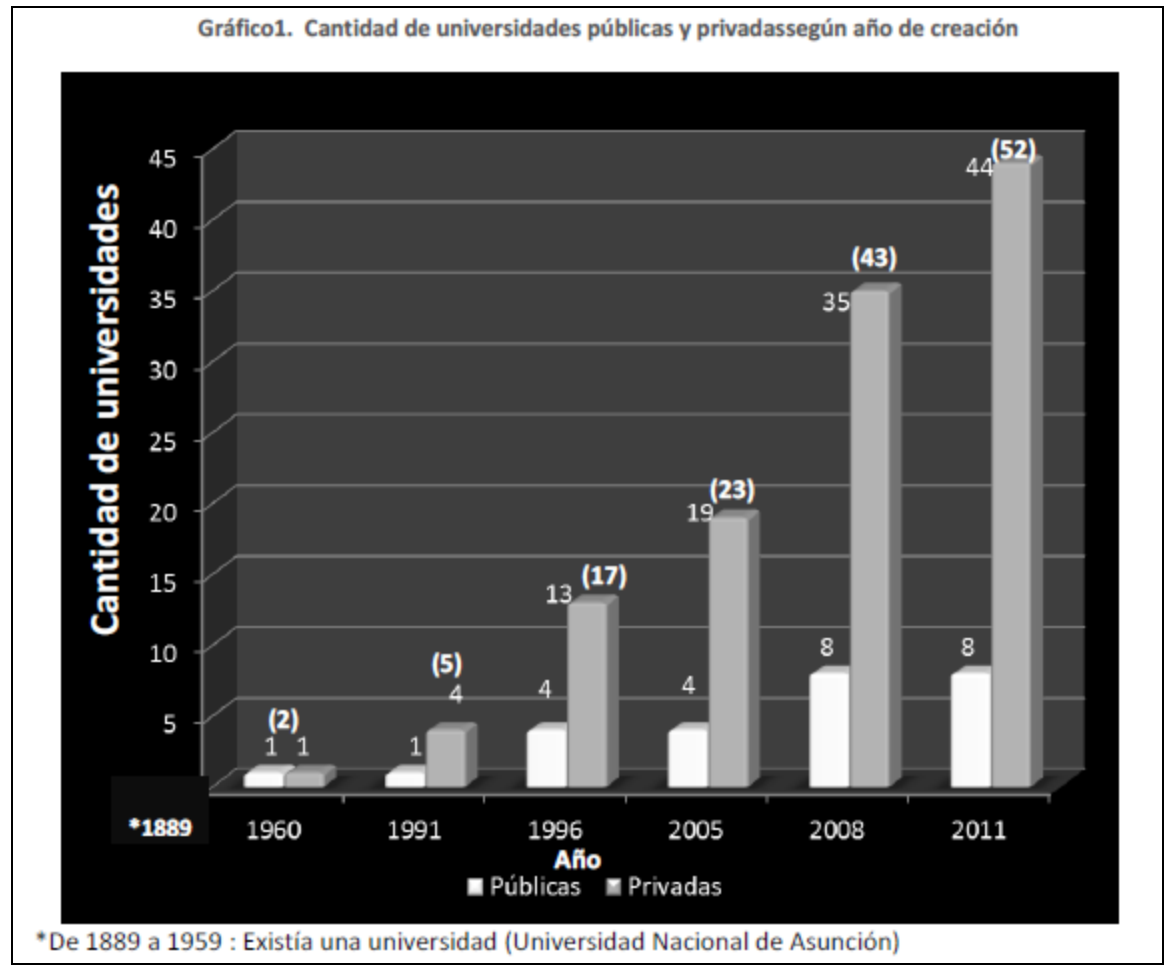

Evolución de la Matricula de grado en las universidades

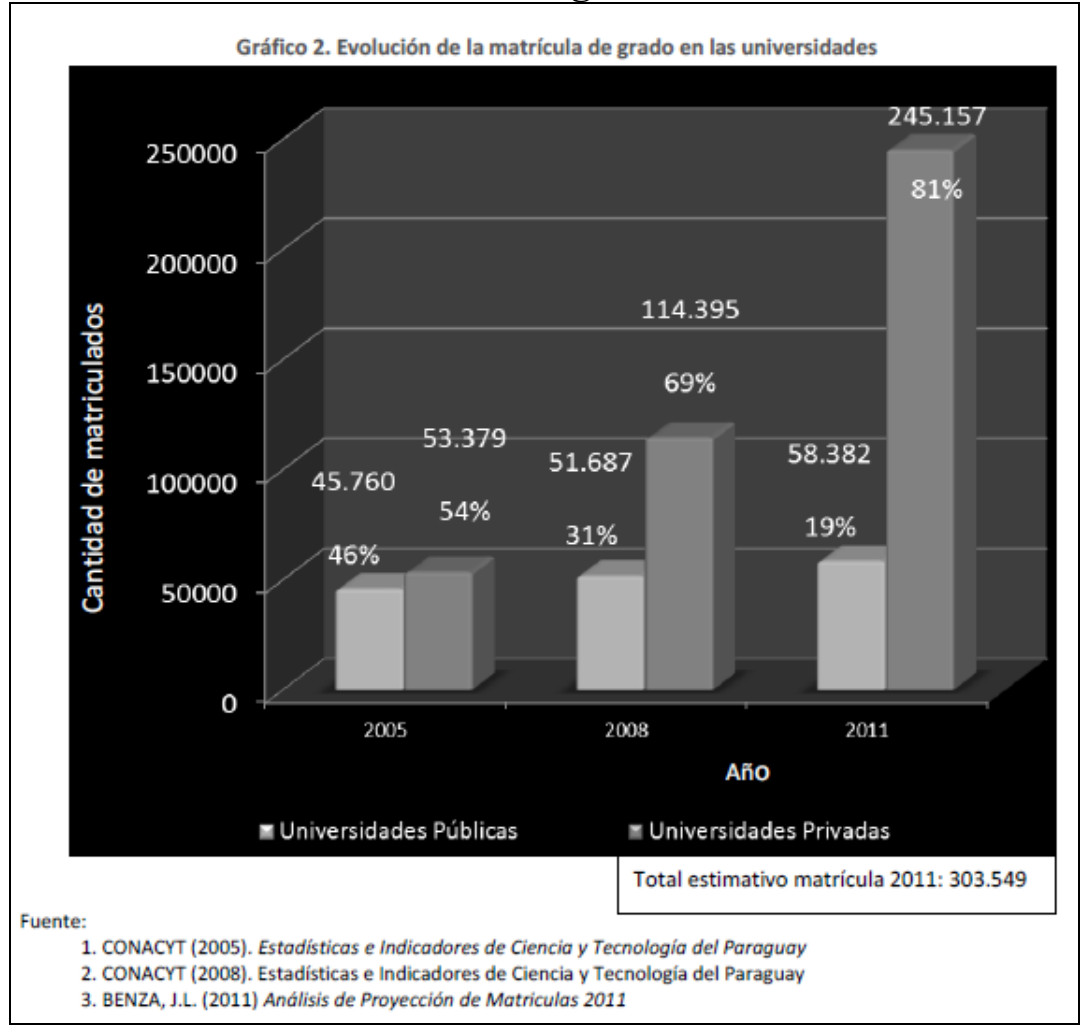




\section{Creación de Institutos de Formación docente}

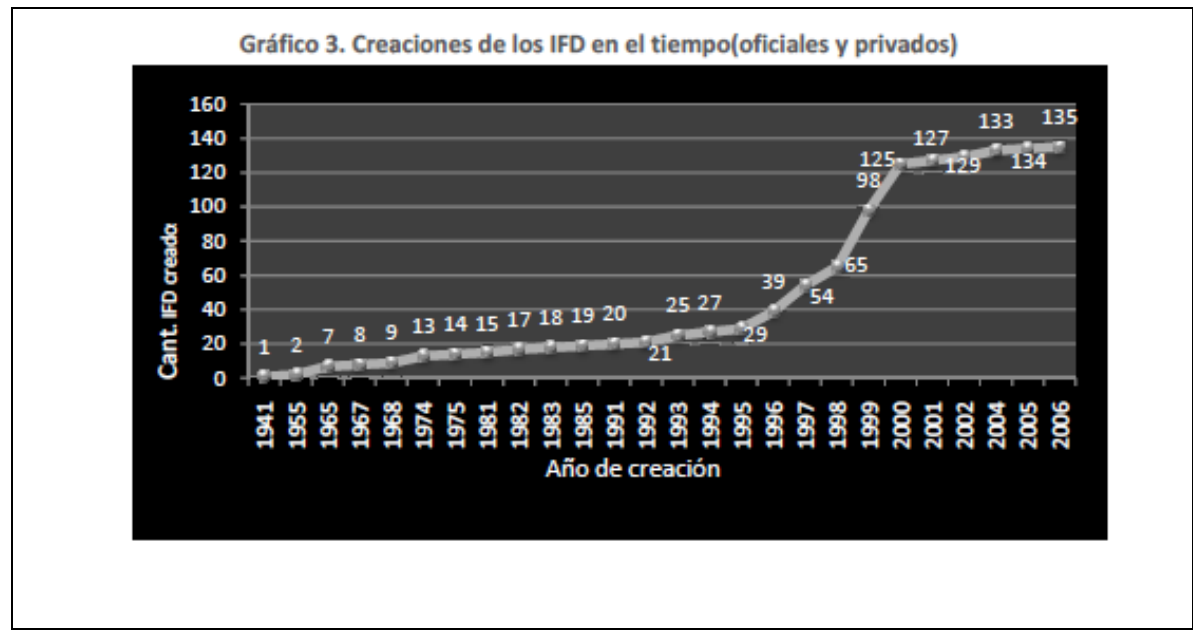

Cuadro comparativo de los IDF según año de creación

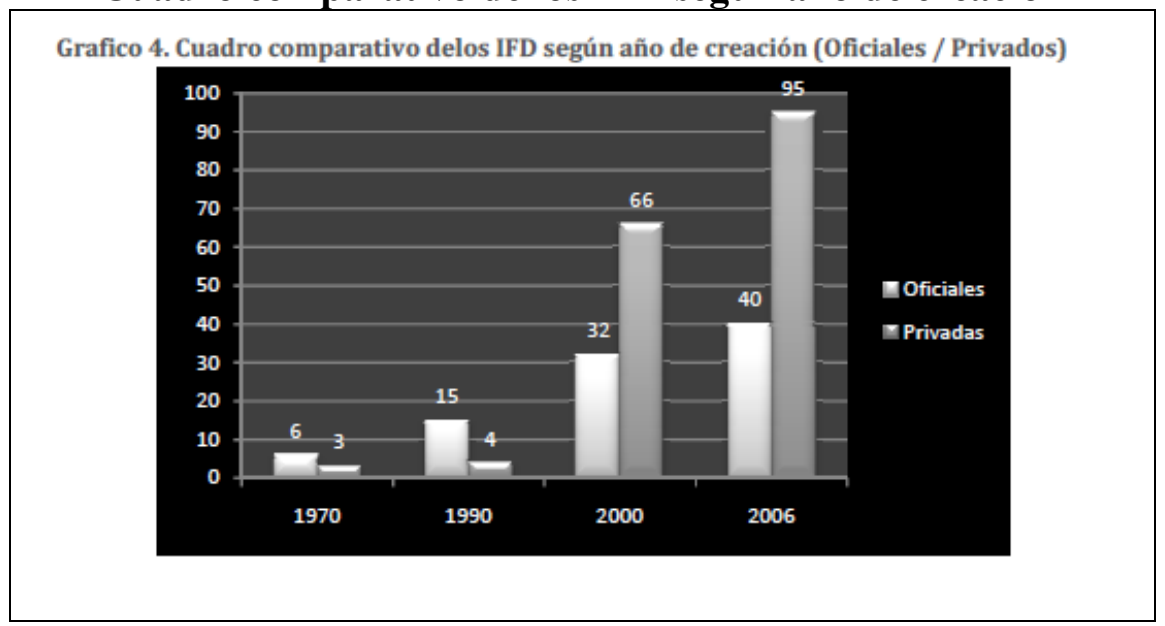

Matriculados por Sector Profesional

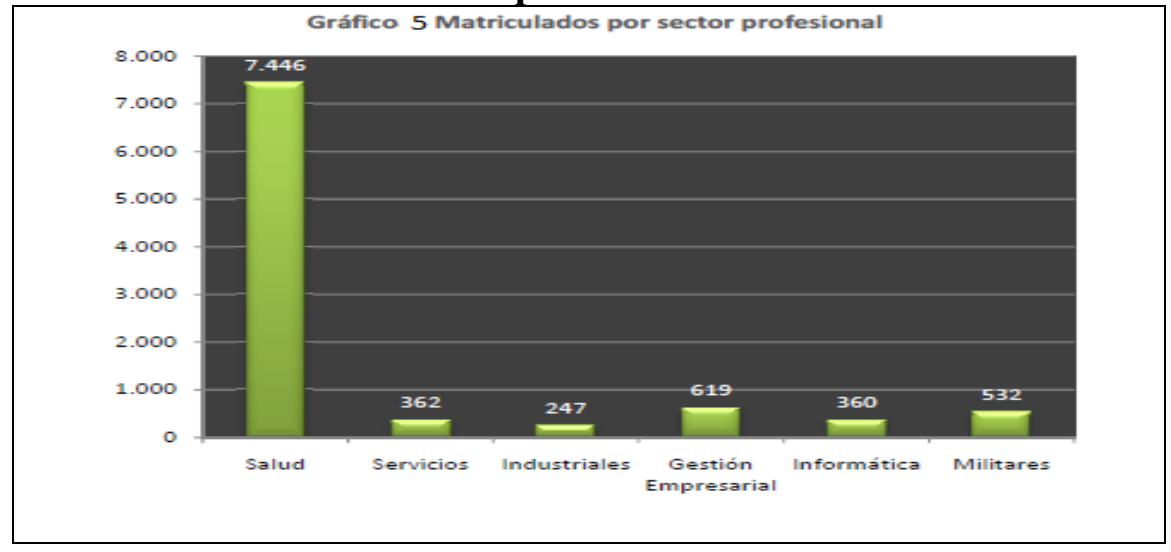

